\title{
Blind I/Q Signal Separation-Based Solutions for Receiver Signal Processing
}

\author{
Mikko Valkama \\ Institute of Communications Engineering, Tampere University of Technology, P.O. Box 553, 33101 Tampere, Finland \\ Email: mikko.e.valkama@tut.fi \\ Markku Renfors \\ Institute of Communications Engineering, Tampere University of Technology, P.O. Box 553, 33101 Tampere, Finland \\ Email: markku.renfors@tut.fi
}

\author{
Visa Koivunen \\ Signal Processing Laboratory, Helsinki University of Technology, P.O. Box 3000, 02015 HUT, Finland \\ Email: visa.koivunen@hut.fi
}

Received 5 February 2004; Revised 14 October 2004

\begin{abstract}
This paper introduces some novel digital signal processing (DSP)-based approaches to some of the most fundamental tasks of radio receivers, namely, channel equalization, carrier synchronization, and I/Q mismatch compensation. The leading principle is to show that all these problems can be solved blindly (i.e., without training signals) by forcing the I and Q components of the observed data as independent as possible. Blind signal separation (BSS) is then introduced as an efficient tool to carry out these tasks, and simulation examples are used to illustrate the performance of the proposed approaches. The main application area of the presented carrier synchronization and I/Q mismatch compensation techniques is in direct-conversion type receivers, while the proposed channel equalization principles basically apply to any radio architecture.
\end{abstract}

Keywords and phrases: radio communications, complex-valued (I/Q) signals and systems, I/Q mismatch, carrier synchronization and tracking, channel equalization, blind signal separation.

\section{INTRODUCTION}

In order to increase the receiver flexibility while also emphasizing the receiver integrability and other implementationrelated aspects, the design of radio receivers is no longer dominated by the traditional superheterodyne architecture. Instead, alternative receiver structures, like the directconversion $[1,2]$ and low-IF $[1,3,4]$ architectures, are receiving more and more interest. The analog front-end of these types of receivers is partially based on complex or I/Q signal processing $[5,6,7]$. More specifically, the frequency translation from radio frequencies (RF) closer to baseband is carried out using I/Q downconversion. Since, in theory, the I/Q downconversion corresponds to a pure frequency translation, the fundamental image signal problem is basically avoided during the downconversion. In this manner, the

This is an open access article distributed under the Creative Commons Attribution License, which permits unrestricted use, distribution, and reproduction in any medium, provided the original work is properly cited. requirements for $\mathrm{RF}$ image rejection filtering are greatly relaxed in practice $[1,2,3,4]$.

The assumption of infinite image signal rejection during the downconversion is strictly valid only if amplitudes and phases of the analog front-end I and Q branches are perfectly matched [7]. In practice, this is not the case. Some mismatch or imbalance will always exist due to imperfections of practical analog electronics. Amplitude imbalances of $1 \%-5 \%$ and phase imbalances of $1-5^{\circ}$ represent feasible design figures $[1,2,3,4]$. This corresponds to approximately 25-40 dB image signal attenuation. These levels of image attenuation are clearly insufficient in low-IF-type receivers where the image band can carry a signal with much higher power level than the desired signal. Some digital signal processing (DSP)-based approaches to improve this image attenuation in IF receivers are presented, for example, in $[7,8,9,10]$. In direct-conversion receivers, the image signal is inherently a self-image (the desired signal itself at negative frequencies), and the analog front-end image attenuation might be sufficient with low-order modulations. 
However, with higher-order modulations, such as 16- or 64-QAM, the distortion due to self-image cannot be neglected and again some kind of compensation is needed $[11,12,13]$. This is also one of the topics of this paper. The idea in this paper is first to show that I/Q mismatch causes crosstalk between the I and Q components. Then this crosstalk or mixing of the I and Q is removed using blind signal separation (BSS) techniques $[14,15,16]$. Compared to the other available solutions in the literature $[12,13]$, the proposed concept is especially attractive since no known training signals are needed. Also the ability to follow possible time dependencies in the mismatch parameters (due to, e.g., temperature changes) is another highly desirable feature.

Another challenging practical problem in radio communications is carrier synchronization [6]. In practice, it is unlikely that the frequency and relative phase of the receiver local oscillators exactly match those of the received carrier. In case of linear modulations, a constant phase offset introduces a constant rotation to the received constellation which needs to be compensated unless differential phase modulation is used. Even a bigger problem is caused by errors or offsets in frequency which basically result in time-varying rotation of the constellation. This is obviously unacceptable for most modulation types and needs to be efficiently compensated for. In this paper, the carrier offsets are shown to result in time-varying leakage between the I and Q, and carrier tracking is implemented with adaptive signal separation methods. Furthermore, when combined with the previous I/Q mismatch compensation, a single BSS stage can accomplish both tasks jointly in a blind manner. These kinds of approaches have not been considered in the literature so far.

In addition to the above front-end-related issues, the distortion due to transmission channel $[6,15]$ is inevitable in any radio receiver and needs to be addressed with care. As will be shown, a general bandpass channel can be viewed to cause frequency-selective crosstalk between the transmitted I and Q data. Based on this, convolutive mixture (or FIR-MIMO) separation techniques working on the observed I and Q signals are applied to implement channel equalization.

The organization of the paper is as follows. The directconversion receiver architecture is shortly reviewed in Section 2, and the basic principles of blind signal separation techniques are presented in Section 3. In Section 4, appropriate MIMO signal models for both I/Q mismatch and carrier offsets are derived, and a signal separation-based approach for blindly compensating these impairments is proposed. Example simulation results are also given to illustrate the efficiency of the proposed concepts. In Section 5, bandpass channel distortion is shown to result in frequency-selective I/Q signal mixing, and, stemming from this analysis, a blind signal separation-based idea to carry out the equalization is formulated. The feasibility of the proposed approach is again verified by computer simulations. Finally, conclusions are drawn in Section 6.

\section{DIRECT-CONVERSION RECEIVER}

\subsection{Background}

The fundamental tasks to be carried out in the front-end of any communications receiver include (i) amplification of the attenuated desired signal, (ii) downconversion of the desired signal spectrum from around the RF carrier frequency closer to baseband, (iii) attenuating the unwanted spectral components appearing in the antenna signal, and (iv) synchronizing the receiver local oscillators for downconversion and sampling with the received signal. Key tasks of the baseband digital signal processing include channel estimation, equalization, and detection. In traditional receiver designs, these tasks are implemented more or less independently of each other, aiming at close-to-ideal operation in each signal processing stage. This has lead to the use of the superheterodyne receiver architecture $[1,4]$ in order to meet the tight RF specifications in terms of image band attenuation and nonlinear effects of the receiver front-end stages. In such receivers, the RF signal is first downconverted to a fixed intermediate frequency (IF) where the receiver selectivity is implemented using a fixed IF filter. The downconversion is usually done with a simple real mixer, and the spectral components on the image signal band need to be attenuated sufficiently by the RF stages before the mixer. Due to the high number of discrete components and high power consumption, the superheterodyne architecture is, however, not the most appropriate choice for highly integrated implementations $[1,2,3,4]$. Furthermore, the use of fixed discrete components in the analog front-end limits the receiver flexibility. Thus, architectures with more simplified analog front-ends with less RF processing are generally needed. In addition, it has recently been demonstrated (see, e.g., [7] and the references therein; see also $[8,9])$ that various nonidealities and distortion effects due to the simplification of the analog front-end can in general be compensated by advanced DSP techniques. This is also the central theme in this paper.

\subsection{Direct-conversion architecture}

A simple way to reduce the number of components in the receiver and alleviate the problem of receiver complexity is to avoid the use of intermediate frequency and quadrature downconvert the desired channel signal directly from RF to baseband. Complete elimination of the IF stage results in highly simplified structure, the so-called direct-conversion receiver, where most of the channel selectivity and amplification are implemented at baseband $[1,2]$. On one hand, since most of the signal processing tasks take place at low frequencies, the power consumption is minimized. On the other hand, very low-noise operation is called for in all the remaining analog components since the amplification provided by the RF stage is only moderate. The direct-conversion receiver concept is depicted in Figure 1.

In the direct-conversion principle, since the IF is effectively zero, the image signal is actually the desired signal itself (at negative center frequency). Ideally, with perfect analog processing, the image band is completely attenuated in 


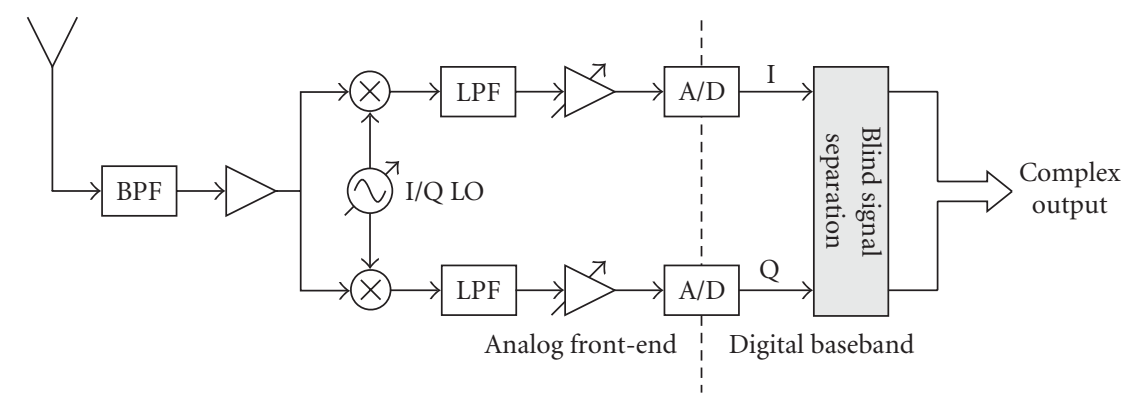

FIGURE 1: The direct-conversion receiver architecture. The leading principle in this paper is to show that various receiver signal processing tasks can be carried out blindly by forcing the observed I and Q signals as independent as possible using blind signal separation.

general. However, practical analog implementations of the needed I/Q signal processing have mismatches in the amplitude and phase responses of the I and Q branches, leading to finite attenuation of the image band signal. In the directconversion case, the effect of imperfect (self-)image rejection is seen as a linear transformation of the original signal constellation $[11,12,13]$. As a result, the image attenuation requirements are not extremely tight, especially if low-order modulations are used. In effect, the $25-40 \mathrm{~dB}$ image attenuation of a practical analog front-end can be sufficient with low-order modulations. With higher-order spectrally efficient modulation methods, however, the distortion due to selfimage can establish an error floor and needs to be compensated.

In practice, the use of zero IF introduces also some other problems. The major drawback in direct-conversion principle is the DC offset problem $[1,2]$. Due to zero IF, the local oscillator frequency is on the same frequency as the desired channel. Then, if the LO signal leaks into the mixer input port, it self-mixes down to baseband causing interfering signal components at zero frequency. These are called DC offsets and can be orders of magnitude larger than the desired channel signal. Besides the LO leakage, another contributor to the offset problems is $1 / f$ noise of the active front-end components. For a satisfactory receiver performance, some compensation of the DC offsets is needed. Another ana$\log$ RF-related problem is that higher linearity is required because in a direct-conversion receiver, second-order intermodulation products may fall in the signal band (in superheterodynes, the weaker third-order intermodulation products usually set the linearity requirements). These problems have limited the use of direct-conversion principle in practical systems earlier, but nowadays this approach is widely utilized in mobile terminals. However, the introduction of higher-order modulations in future wireless communication systems sets higher demands for the receiver performance, and the I/Q imbalance effects, for example, to be discussed in Section 4 are likely to pose big challenges.

\section{BLIND SIGNAL SEPARATION}

Currently, more and more applications call for proper representation of multivariate data. An extension of principal component analysis (PCA) called independent component analysis (ICA) is one good example of such techniques [16]. The ICA and its signal processing application, blind signal separation $[14,16]$, is applied in this paper to communications receiver signal processing. The purpose of this chapter is to introduce the basic concepts and notations after which the actual applications to I/Q mismatch and carrier offset compensation as well as to channel equalization are discussed and analyzed in Sections 4 and 5.

Generally speaking, blind signal separation deals with the recovery of some interesting signals, called sources, based on observing their linear ${ }^{1}$ mixtures, and falls under the umbrella of multiple-input multiple-output (MIMO) signal processing $[14,15,16]$. The term blind is used here to emphasize the fact that no prior knowledge of the mixing process or the temporal structure of the underlying source signals is needed, but only the statistical properties are utilized. The leading principle in this context is the assumption of statistical independence of the original sources $[14,15,16]$. Thus in practice the recovery consists of forcing the separator output signals to be "as independent as possible," according to the selected independence measure. Commonly used approaches in this context are, for example, nonlinear decorrelation and minimization of mutual information. As is obvious, the relative order of the recovered sources or the individual amplitude/power levels cannot be blindly identified.

In addition to statistical independence, another key assumption is that only one of the sources (if any) can be Gaussian $[14,15,16,17]$. This is easy to understand since for Gaussian signals uncorrelatedness implies independence, making it impossible to distinguish any (remaining) orthogonal/orthonormal transformation. Luckily most communications signals are, indeed, non-Gaussian. In the following, $M$ and $N$ denote the number of observed and original source signals, respectively.

\subsection{Instantaneous or frequency-nonselective MIMO models}

Assuming the mixing process is instantaneous, the $m$ th observation, say $x_{m}(k)$, is of the form $x_{m}(k)=a_{m, 1} s_{1}(k)+$ $a_{m, 2} s_{2}(k)+\cdots+a_{m, N} s_{N}(k)$, where $s_{n}(k)$ denotes the $n$th

\footnotetext{
${ }^{1}$ Also nonlinear BSS techniques have been proposed; see, for example, [16] and the references therein. The techniques applied in this paper are, however, based on linear signal models.
} 
source signal and $a_{m, n}$ represents the relative weight of the source $s_{n}(k)$ in the observation $x_{m}(k)$. Stacking the observed and source signal samples at time-instant $k$ into column vectors $\mathbf{s}(k)=\left[s_{1}(k), s_{2}(k), \ldots, s_{N}(k)\right]^{T}$ and $\mathbf{x}(k)=$ $\left[x_{1}(k), x_{2}(k), \ldots, x_{M}(k)\right]^{T}$, respectively, the system model can be simply written as

$$
\mathbf{x}(k)=\mathbf{A s}(k),
$$

where the $M \times N$ matrix A describes the mixing process with $[\mathbf{A}]_{m n}=a_{m, n}$. In general, the matrix $\mathbf{A}$ is assumed unknown but nonsingular (full rank). This is a natural assumption and is fundamental for the identifiability of the model; see [14, $16,17,18]$ for more details.

Generally speaking, the separator processes a sequence of the observation vectors and tries to "invert" the model in (1). In adaptive separation, the parameters of the separator are updated iteratively which also enables tracking the possible time-variant features of the mixture model. The separator output at time $k$ is commonly written as

$$
\mathbf{y}(k)=\mathbf{W}(k) \mathbf{x}(k)=\mathbf{W}(k) \mathbf{A s}(k)=\mathbf{T}(k) \mathbf{s}(k),
$$

where $\mathbf{W}(k)$ denotes the $N \times M$ separator matrix, $\mathbf{T}(k)=$ $\mathbf{W}(k) \mathbf{A}$ is the total equivalent system matrix $(N \times N)$, and $\mathbf{y}(k)=\left[y_{1}(k), y_{2}(k), \ldots, y_{N}(k)\right]^{T}$. For successful separation, $\mathbf{T}(k)$ should converge to a "quasi-identity" (permutation and scaling) matrix with only one nonzero element on each of its row and column.

Various algorithms, with varying computational complexity and performance, to determine the separator coefficients exist in the literature; see $[14,16]$ for excellent reviews. One exciting feature of the separation stage is the possibility for uniform performance. This being the case, the separation performance is independent of the underlying mixture coefficients (i.e., the matrix $\mathbf{A}$, as long as it is full rank) and depends only on the source statistics. One such algorithm with this desirable property is the so-called equivariant adaptive separation via independence (EASI), proposed originally in [19], whose performance depends only on the certain nonlinear moments of the source signals. The EASI algorithm consists formally of two subtasks; a whitening (secondorder decorrelation) part and a nonlinear decorrelation part where the selection of the used nonlinear function depends on the source statistics. The exact algorithm description can be found in [19]. This algorithm is used also in this paper in Section 4. Notice, however, that this is done only to illustrate the principal operation of the proposed receiver concepts; thus any other adaptive separation approach could be tested and used as well in practice.

\subsection{Convolutive or frequency-selective MIMO models}

A more general class of signal models is obtained if the assumption of instantaneous mixing is dropped. In other words, the mixing process can also contain memory and thus be frequency selective $[14,16,20]$. A direct extension of the model in (1) results in

$$
\mathbf{x}(k)=\sum_{l} \mathbf{A}_{l} \mathbf{s}(k-l)
$$

which represents a MIMO convolution of the sequences $\{\ldots, \mathbf{s}(k-1), \mathbf{s}(k), \mathbf{s}(k+1), \ldots\}$ and $\left\{\ldots, \mathbf{A}_{-1}, \mathbf{A}_{0}, \mathbf{A}_{1}, \ldots\right\}$, with each $\mathbf{A}_{l}$ being size $M \times N$. In other words, each observation $x_{n}(k)$ is a convolutive mixture of the original source signals. In this case, the identifiability condition related to the mixing process is generally formulated in terms of the corresponding system (MIMO) transfer function $\mathbf{A}(z)=\sum_{l} \mathbf{A}_{l} z^{-l}$; the recovery of the original source contributions is feasible if this system transfer function has full rank $[14,16,20]$.

Practical recovery of the source signals consists of multichannel filtering of the observed vectors and can generally be written as

$$
\mathbf{y}(k)=\sum_{l} \mathbf{W}_{l}(k) \mathbf{x}(k-l)
$$

where the $N \times M$ separator matrices $\mathbf{W}_{l}(k)$ are adapted to minimize the predetermined dependence measure between the components of $\mathbf{y}(k)$. In terms of system transfer functions, the relation between the source signals and the separator output signals is of the form

$$
\mathbf{T}(z, k)=\mathbf{W}(z, k) \mathbf{A}(z)
$$

where $\mathbf{W}(z, k)$ is the transfer function of the separator at time $k$. For successful source recovery, the total system $\mathbf{T}(z, k)$ should converge to a matrix with only one non-zero element in each row and column.

As in case of instantaneous mixtures, also here a wide variety of different algorithms for separator adaptation exists in the literature, and some of them can be claimed to have the uniform separation performance $[14,16,20]$. One example is the natural gradient-based approach described in [20]. This algorithm is applied also in this paper in Section 5 to the channel equalization problem in terms of the I and Q signals.

\section{BLIND I/Q MISMATCH AND CARRIER OFFSET COMPENSATION}

In this section, the I/Q mismatch problem due to analog front-end nonidealities as well as the carrier synchronization task are addressed in detail for direct-conversion receivers. The basic idea is to show that both of these practical problems can be viewed to create dependence between the observed I and Q signals. Then, a signal separation algorithm is applied to remove this dependence and thus to recover the original I and Q data. 


\subsection{Signal models and $I / Q$ separation-based compensation}

\subsubsection{I/Q mismatch}

For analysis purposes, the received RF signal, say $r(t)$, is written as

$$
\begin{aligned}
r(t) & =\operatorname{Re}\left[z(t) \exp \left(j \omega_{C} t\right)\right] \\
& =z_{I}(t) \cos \left(\omega_{C} t\right)-z_{Q}(t) \sin \left(\omega_{C} t\right),
\end{aligned}
$$

where $z(t)=z_{I}(t)+j z_{Q}(t)$ denotes the corresponding ideal baseband equivalent of the desired channel to be recovered by the receiver front-end. Now, to model the amplitude and phase mismatches of the analog front-end, the (complex) LO signal of the I/Q downconverter is written as

$$
\begin{gathered}
x_{\mathrm{LO}}(t)=\cos \left(\omega_{C} t\right)-j g \sin \left(\omega_{C} t+\phi\right) \\
=K_{1} \exp \left(-j \omega_{C} t\right)+K_{2} \exp \left(j \omega_{C} t\right), \\
K_{1}=\frac{1+g \exp (-j \phi)}{2}, \quad K_{2}=\frac{1-g \exp (j \phi)}{2},
\end{gathered}
$$

where $g$ and $\phi$ represent the relative amplitude and phase mismatches, respectively. The latter form of (7) indicates that two frequency translations take place due to mismatches. Indeed, the downconversion of $r(t)$ combined with lowpass filtering results in

$$
x(t)=K_{1} z(t)+K_{2} z^{*}(t)
$$

and the corresponding self-image rejection ratio is $10 \log _{10}\left(\left|K_{1}\right|^{2} /\left|K_{2}\right|^{2}\right)$.

To examine the mismatch effect from the I and Q signal point of view, the model in (9) can be written as $x(t)=$ $x_{I}(t)+j x_{Q}(t)$, where

$$
\begin{gathered}
x_{I}(t)=z_{I}(t), \\
x_{Q}(t)=g \cos (\phi) z_{Q}(t)-g \sin (\phi) z_{I}(t) .
\end{gathered}
$$

In other words, I/Q mismatch tends to create dependence between the I and Q signals. Assuming that the original I and Q signals are statistically independent, which holds, for example, for square QAM type of constellations, these original I and Q components can be recovered blindly using a signal separation algorithm. More explicit formulation is given in Section 4.1.3.

\subsubsection{Carrier offsets}

To see the explicit effect of carrier offsets more formally, the I/Q downconverter LO signal is now written as

$$
\begin{aligned}
x_{\mathrm{LO}}(t) & =\cos \left(\left(\omega_{C}+\Delta \omega\right) t+\theta\right)-j \sin \left(\left(\omega_{C}+\Delta \omega\right) t+\theta\right) \\
& =\exp \left(-j\left(\left(\omega_{C}+\Delta \omega\right) t+\theta\right)\right),
\end{aligned}
$$

where $\Delta \omega$ and $\theta$ model the frequency and phase offsets, respectively, relative to the received signal in (6). Now, it is common to write the downconverted signal after lowpass filtering as

$$
x(t)=z(t) \exp (-j(\Delta \omega t+\theta)) .
$$

Interestingly, when written in terms of the I and Q signals, the model in (12) can be expressed as

$$
\begin{aligned}
x_{I}(t) & =\cos (\Delta \omega t+\theta) z_{I}(t)+\sin (\Delta \omega t+\theta) z_{Q}(t), \\
x_{Q}(t) & =\cos (\Delta \omega t+\theta) z_{Q}(t)-\sin (\Delta \omega t+\theta) z_{I}(t) .
\end{aligned}
$$

Thus, from the I/Q point of view, the carrier offsets correspond to time-varying mixing of the I and Q signals, and an adaptive signal separation algorithm can be used to track and remove this effect.

A combined signal model incorporating both the I/Q mismatch and the carrier offset effects is given next. Using analysis similar to those given above, it is relatively easy to show that observable signal after downconversion and lowpass filtering appears as

$$
\begin{aligned}
x(t)= & K_{1} z(t) \exp (-j(\Delta \omega t+\theta)) \\
& +K_{2} z^{*}(t) \exp (j(\Delta \omega t+\theta)) .
\end{aligned}
$$

Here it is naturally assumed that the frequency offset is smaller than the guard band between the adjacent frequency channels. Now, the complex signal in (14) corresponds to an I/Q signal pair of the form

$$
\begin{gathered}
x_{I}(t)=\cos (\Delta \omega t+\theta) z_{I}(t)+\sin (\Delta \omega t+\theta) z_{Q}(t), \\
x_{Q}(t)=g \cos (\Delta \omega t+\theta+\phi) z_{Q}(t)-g \sin (\Delta \omega t+\theta+\phi) z_{I}(t) .
\end{gathered}
$$

\subsubsection{Joint compensation using blind I/Q signal separation}

As given in (15), the observable I and Q signals in the presence of I/Q mismatch and carrier offsets appear as instantaneous and time-varying mixtures of the true I and $\mathrm{Q}$ signals. Switching to discrete-time notations $x_{I}(k) \equiv x_{I}\left(k T_{S}\right)$, and so forth, we introduce $2 \times 1$ source and observation vectors $\mathbf{s}(k)=\left[z_{I}(k), z_{Q}(k)\right]^{T}$ and $\mathbf{x}(k)=\left[x_{I}(k), x_{Q}(k)\right]^{T}$ and write the model in (15) as $\mathbf{x}(k)=\mathbf{A}(k) \mathbf{s}(k)$, where

$$
\mathbf{A}(k)=\left[\begin{array}{cc}
\cos (\Delta \omega k+\theta) & \sin (\Delta \omega k+\theta) \\
-g \sin (\Delta \omega k+\theta+\phi) & g \cos (\Delta \omega k+\theta+\phi)
\end{array}\right] .
$$

Notice that the frequency offset $\Delta \omega$ refers here to the "normalized" frequency variable $\Delta \omega=2 \pi \Delta f / f_{S}$. Now an adaptive signal separation algorithm, such as the EASI algorithm discussed in Section 3, can be used to blindly estimate the source signals $z_{I}(k)$ and $z_{Q}(k)$. 
It is interesting to note that the identifiability of the model in (16) is independent of the carrier offset levels and also practically independent of the mismatch values. This can be seen more formally by examining the determinant of $\mathbf{A}(k)$ :

$$
\begin{aligned}
\operatorname{det}(\mathbf{A}(k))= & g \cos (\Delta \omega k+\theta) \cos (\Delta \omega k+\theta+\phi) \\
& +g \sin (\Delta \omega k+\theta) \sin (\Delta \omega k+\theta+\phi) \\
= & g \cos (\phi)
\end{aligned}
$$

Thus, the system matrix $\mathbf{A}(k)$ is invertible given that $g \neq 0$ and $\phi \neq \pm \pi / 2\left( \pm 90^{\circ}\right)$. The first requirement $(g \neq 0)$ simply states that the downconversion stage needs to produce two non-zero signals while the second one prevents the case where the two signals after downconversion would be just scaled versions of each other. These are more than natural requirements for any I/Q front-end and are always fulfilled by any practical analog design. Thus this indicates that the proposed idea is robust in the face of different imbalance and offset levels in terms of identifiability.

There are some further practical issues related to the proposed compensation scheme. First of all, as discussed in Section 2, the direct-conversion architecture suffers from the well-known DC offset problem [1,2,4] due to self-mixing of the LO signal leaking into the mixer RF port. Most signal separation algorithms, in turn, assume zero-mean data, so the DC offset needs to be compensated prior to the separation stage. Another practical aspect is related to the amount of frequency offsets tolerated. On one hand, the frequency offset should be smaller than the guard band between adjacent frequency channels. If not, the receiver is not anymore really zero IF but closer to low IF and the nearby channel signal (or at least part of it located on the true image band) appears as interference on top of the desired signal after downconversion. On the other hand, the frequency offset determines the dynamics of the system matrix $\mathbf{A}(k)$ in (16), which is indeed the dynamics that the adaptive separation algorithm needs to follow. In other words, this dynamics should be within the tracking capability of the applied adaptive algorithm. Commonly, this poses some limitations to the used step-sizes such that a relatively large stepsize is needed. The used step-size, in turn, is usually directly related to the separator steady-state performance and cannot, of course, exceed its own algorithm-specific stability limit (see, e.g., $[14,16,19]$ for more details). Thus we can conclude that even though the frequency offset level is irrelevant from the identifiability point of view, the tracking capability of the practical algorithms as well as the role of the image signal limit the applicability of the proposed concept to mild frequency offset. In other words, coarse frequency synchronization should be implemented by other means prior to the separation stage. Notice also that due to the amplitude (sign) and ordering ambiguities mentioned in Section 3, it is possible that the recovered constellation still formally suffers from (i) a constant phase rotation of (integer multiple of) $90^{\circ}$ and/or (ii) complex conjugation. In practice, these issues can be easily resolved using a little side information in the actual data detection stage. It should be noted that any

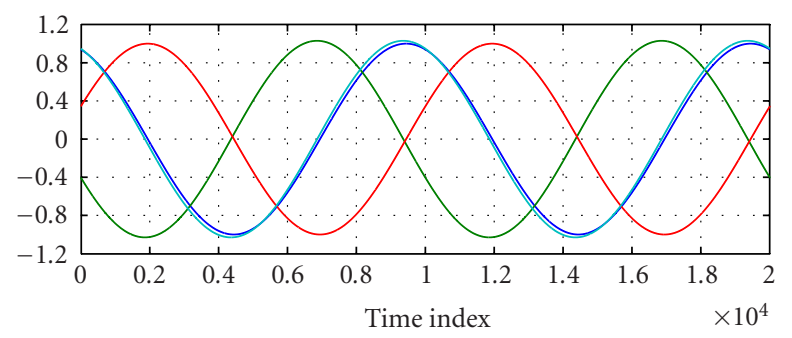

(a) Mixture coefficients.

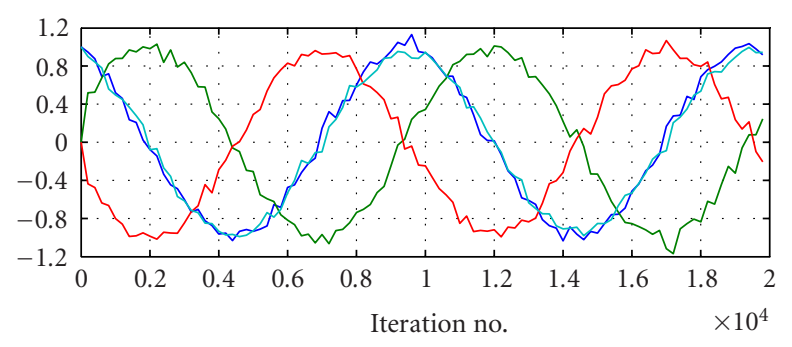

(b) Separator coefficients.

FIgure 2: (a) An illustration of the dynamics of the system matrix. (b) One realization of the separator coefficients using the EASI algorithm (step-size 0.01). The I/Q mismatch values: $g=1.03$ and $\phi=3^{\circ}$. The carrier offset levels: $\theta=20^{\circ}$ and $\Delta \omega=2 \pi \times 0.0001$. Additive noise SNR $=20 \mathrm{~dB}$.

blind algorithm is subject to similar ambiguities in general. As mentioned above, these ambiguities can be reduced in a later stage of the receiver, for example, by using a minimal number of known symbols (pilot or training symbols specified in the signaling frame structure) or by using differential coding/mapping between bits and symbols. In general, for modulations other than square QAM, the effects of possible dependence between the true I and Q should be explored individually.

\subsection{Simulation example}

Here some example results are given to illustrate the efficiency of the proposed compensation idea. In the simulations, imbalance levels of $3 \%$ and $3^{\circ}$ are used corresponding to an approximate of $30 \mathrm{~dB}$ image attenuation which should represent a typical practical case. Phase offset in the system is assumed to be $20^{\circ}$ and the (remaining) frequency offset $0.0001 \times 2 \pi$. Given, for example, a $10 \mathrm{MHz}$ sampling frequency, this corresponds to $1 \mathrm{kHz}$ absolute frequency offset. The actual data modulation is 16 QAM. The model also includes additive white Gaussian noise (AWGN) with the signal-to-noise ratio (SNR) ranging from $0 \mathrm{~dB}$ to $20 \mathrm{~dB}$. The EASI algorithm discussed in Section 3 is then used as an example algorithm in the separation stage with a step-size of 0.01 and a third-order (cubic) nonlinearity [19].

The time-varying mixture coefficients are illustrated in Figure 2a, followed below by an example realization of the separator coefficients in Figure $2 b$ with SNR of $20 \mathrm{~dB}$. Clearly, the separation algorithm is able to track the 


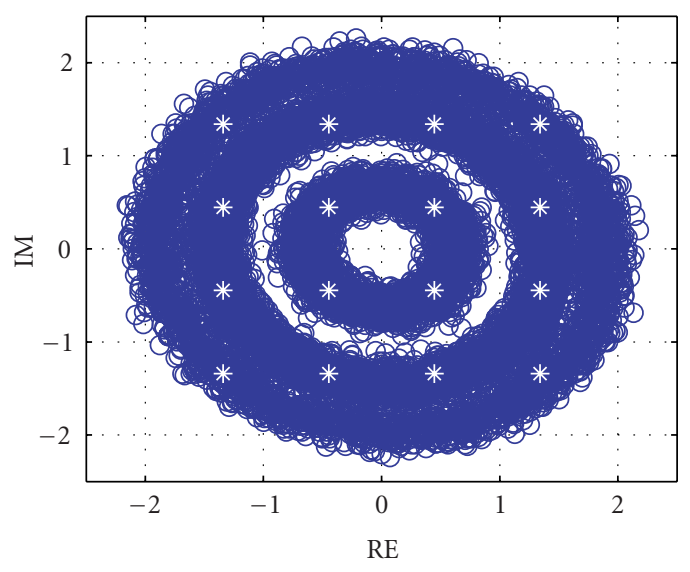

(a) Without compensation.

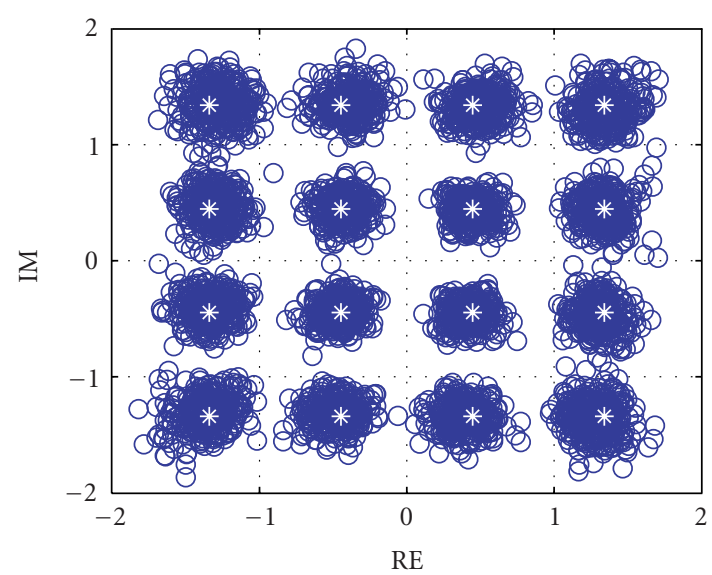

(b) With compensation.

FIGURE 3: Symbol rate output samples (16-QAM) (a) without and (b) with compensation. The I/Q mismatch values: $g=1.03$ and $\phi=3^{\circ}$. The carrier offset levels: $\theta=20^{\circ}$ and $\Delta \omega=2 \pi \times 0.0001$. Additive noise $\mathrm{SNR}=20 \mathrm{~dB}$.

time-varying mixture coefficients successfully. The corresponding symbol rate output samples without and with compensation are depicted in Figure 3. As is evident, the signal without compensation is useless due to I/Q mismatch and carrier offsets. The compensator output signal, however, is a good estimate of the transmitted symbol constellation.

The most fundamental performance measure of any digital communication system is the bit or symbol error rate (BER/SER). This is assessed next for the proposed compensator as a function of additive noise SNR. The decisions are made symbol by symbol using the minimum distance detection principle. The obtained results are depicted in Figure 4 which also shows the SER with additive noise only for reference. The corresponding SER without any compensation is close to one, independently of the SNR, and is not shown for simplicity. As is evident, the proposed compensator can efficiently estimate the transmitted signal, bringing the error rate close to the AWGN bound. Especially in the raw SER

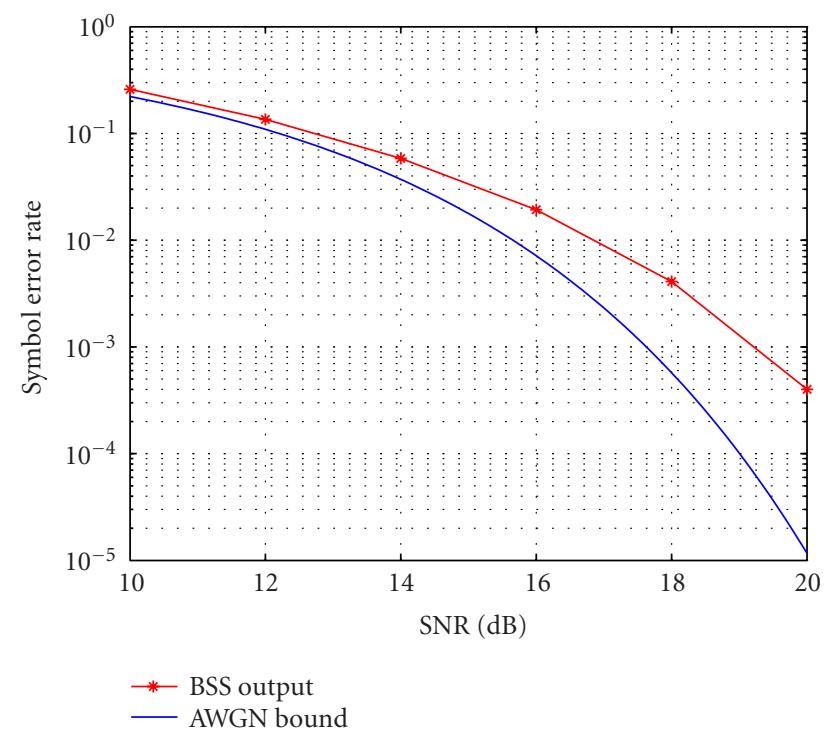

FIgURE 4: Symbol error rate of the EASI algorithm-based compensator for 16-QAM data. The I/Q mismatch values: $g=1.03$ and $\phi=3^{\circ}$. The carrier offset levels: $\theta=20^{\circ}$ and $\Delta \omega=2 \pi \times 0.0001$. Also shown for reference is the symbol error rate with additive noise only (AWGN bound).

levels of $10^{-1}$ to $10^{-2}$, which is the crucial operating range of any practical system before error-control decoding, the proposed receiver is really close (within $1 \mathrm{~dB}$ ) to the noise limit.

\section{BLIND CHANNEL EQUALIZATION}

In this section, the traditional channel equalization problem is addressed from the I/Q signal processing point of view. The leading idea is to show that a typical bandpass channel encountered in radio communications results in frequencyselective mixing of the input I and Q signals. Then, a convolutive mixture separation algorithm is applied to blindly recover the transmitted data.

\subsection{Signal models and $I / Q$ separation-based blind equalization}

The starting point in the following is the traditional baseband-equivalent system model [6] commonly written as

$$
x(k)=h(k) * z(k)+v(k),
$$

where $z(k)$ denotes the transmitted data sequence, $h(k)$ is the baseband-equivalent impulse response of the true bandpass channel, $v(k)$ represents additive noise, and $*$ denotes convolution. In general, all the variables are complex valued. The task of an equalizer is then to recover the transmitted sequence $z(k)$ using only the observed signal $x(k)$ and any available side information such as the input signal statistics. 
The model in (18) can also be written in terms of the I and Q signals as $x(k)=x_{I}(k)+j x_{Q}(k)=\left(h_{I}(k)+j h_{Q}(k)\right) *$ $\left(z_{I}(k)+j z_{Q}(k)\right)+v_{I}(k)+j v_{Q}(k)$ or

$$
\begin{aligned}
& x_{I}(k)=h_{I}(k) * z_{I}(k)-h_{Q}(k) * z_{Q}(k)+v_{I}(k), \\
& x_{Q}(k)=h_{Q}(k) * z_{I}(k)+h_{I}(k) * z_{Q}(k)+v_{Q}(k) .
\end{aligned}
$$

In other words, $x_{I}(k)$ and $x_{Q}(k)$ both appear as convolutive mixtures of the transmitted data $z_{I}(k)$ and $z_{Q}(k)$. As a result, blind equalization can also be viewed as a blind I/Q signal separation task with $2 \times 1$ source and observation vectors $\mathbf{s}(k)=\left[z_{I}(k), z_{Q}(k)\right]^{T}$ and $\mathbf{x}(k)=\left[x_{I}(k), x_{Q}(k)\right]^{T}$, assuming that the I and Q components of the transmitted data are again independent. The corresponding $2 \times 2$ system transfer function matrix is given by

$$
\mathbf{A}(z)=\left[\begin{array}{cc}
H_{I}(z) & -H_{Q}(z) \\
H_{Q}(z) & H_{I}(z)
\end{array}\right],
$$

where $H_{I}(z)$ and $H_{Q}(z)$ denote the $z$-transforms of $h_{I}(k)$ and $h_{Q}(k)$, respectively. Considering the identifiability of the above model, the determinant of $\mathbf{A}(z)$ can be expressed as

$$
\operatorname{det}(\mathbf{A}(z))=H_{I}^{2}(z)+H_{Q}^{2}(z)
$$

Thus the model is strictly identifiable if $H_{I}^{2}\left(e^{j \omega}\right)+H_{Q}^{2}\left(e^{j \omega}\right) \neq$ 0 for all $\omega$ within the bandwidth of $z(k)$. Notice that this condition is not exactly identical to $\left|H\left(e^{j \omega}\right)\right|^{2}$ being non-zero. Obviously, as in any other BSS task, the relative order and amplitudes (signs) of the sources (I and Q) cannot be blindly identified.

Based on the model in (20), also the $2 \times 2$ separator transfer matrix $\mathbf{W}(z)$ has only two unknown elements (polynomials of $z$ ). This information can be used to simplify the separation algorithm. Assuming that the elements of $\mathbf{W}(z)$ are FIR (finite impulse response) filters of order $L$, this reduces the amount of unknowns from the general case of $4 \times(L+1)$ to only $2 \times(L+1)$, and the actual adaptation algorithm, such as the natural gradient-based algorithm discussed in Section 3, can be simplified accordingly.

It should be noted that any equalizer filter, say $w^{\mathrm{eq}}(k)=$ $w_{I}^{\mathrm{eq}}(k)+j w_{Q}^{\mathrm{eq}}(k)$, can be interpreted as a $2 \times 2$ separating system

$$
\mathbf{W}^{\mathrm{eq}}(z)=\left[\begin{array}{cc}
W_{I}^{\mathrm{eq}}(z) & -W_{Q}^{\mathrm{eq}}(z) \\
W_{Q}^{\mathrm{eq}}(z) & W_{I}^{\mathrm{eq}}(z)
\end{array}\right]
$$

However, up to our knowledge, none of the blind equalization techniques presented in the literature acknowledges this fact or takes advantage of it in the design. The connection in general between the blind signal separation and blind channel equalization or deconvolution is discussed in

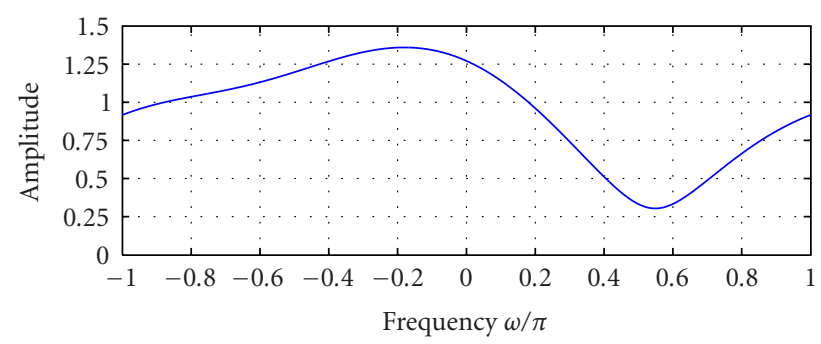

Figure 5: Amplitude response of the baseband-equivalent channel $H(z)=H_{1}(z)+j H_{Q}(z)$ used in the simulation.

the literature, for example, $[14,15,16,21]$, but the formulation given in this paper using the I/Q separation approach has not been considered so far. In general, the solutions relating blind deconvolution and blind signal separation call for input data with independent samples in time. Notice that this assumption is not needed here, but the I and Q components of the input data were assumed independent. Notice also that the complexity of the signal model is here significantly lower (only $2 \times 2$ ) compared to other approaches discussed in $[14,15,16,21]$.

\subsection{Simulation example}

Some example results of computer simulations are given next to illustrate the efficiency of the proposed equalizer concept. A 16-QAM data modulation with independent I and Q components $\left(z_{I}(k)\right.$ and $\left.z_{Q}(k)\right)$ is used, and the transmitted signal travels through an example baseband-equivalent channel whose amplitude response is depicted in Figure 5. The "notch" in the used channel is around $13 \mathrm{~dB}$ below the maximum amplitude response level. As can easily be verified, this channel fulfills the identifiability condition mentioned in Section 5.1. The system also includes additive white Gaussian noise (AWGN), with the signal-to-noise ratio (SNR) being set to $20 \mathrm{~dB}$ in this experiment. The I and Q samples of the channel distorted noisy signal (see Figure 6a) are then processed using the natural gradient-based separation algorithm [20] with nine-tap FIR separation filters. A third-order nonlinearity is used in the algorithm and the step-size is 0.001 . With these selections, it takes around 5000 iterations to reach the steady state in a stationary environment.

The first two components $T_{1,1}(z)$ and $T_{1,2}(z)$ (with $\left.T_{m, n}(z)=[\mathbf{T}(z)]_{m n}\right)$ of the total equivalent system $\mathbf{T}(z)=$ $\mathbf{W}(z) \mathbf{A}(z)$ are depicted in the steady state in terms of their amplitude responses in Figure 7 . The other two are not shown due to symmetry (i.e., $T_{2,1}(z)=-T_{1,2}(z)$ and $\left.T_{2,2}(z)=T_{1,1}(z)\right)$. Obviously, the first output $y_{1}(k)$ forms a good estimate of $z_{I}(k)$ and the other output $y_{2}(k)$ of $z_{Q}(k)$, since the crosstalk between I and Q is around $30 \mathrm{~dB}$ below the target signal level. In other words, $y_{1}(k)+j y_{2}(k)$ is a good estimate of the transmitted complex data and the channel equalization is successful. This is further illustrated in Figure 8 in terms of the effective error norm between the total equivalent impulse response of the equalized system (channel + separator) and a pure impulse as a function of 


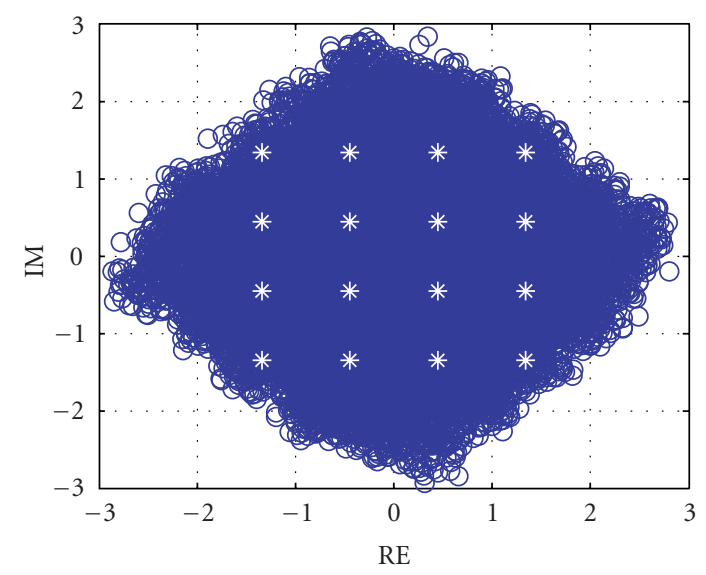

(a)

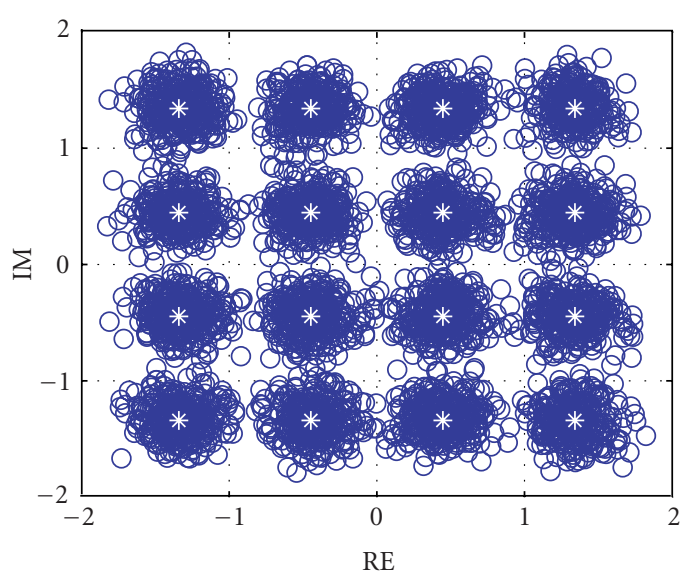

(b)

Figure 6: Symbol rate received samples (16-QAM) (a) without and (b) with I/Q signal separation-based equalization. Additive noise SNR is $20 \mathrm{~dB}$.

iteration number. As can be seen, the total impulse response really converges to an impulse (or a delayed impulse in general), verifying successful equalization. The symbol rate output samples without and with I/Q signal separator are depicted in Figure 6.

Next we assess the symbol error rate (SER) performance of the proposed equalizer, considering the given channel and varying the SNR from $10 \mathrm{~dB}$ to $20 \mathrm{~dB}$. For reference purposes, the observed signal is also processed by a traditional trainingbased equalizer $[6,22]$ whose coefficients (also a nine-tap complex FIR filter) are adapted using the well-known leastmean-square (LMS) algorithm [22]. The resulting symbol error rates for both equalizers are depicted in Figure 9, evidencing surprisingly similar performance from the SER point of view. The BSS-based solution, however, does not need any training data and thus results in increased effective user data rates.

Generally speaking, it should be noted that the proposed I/Q separation-based equalizer, as any linear equalizer, suffers from the noise enhancement in channels with deep notches within the signal band. This is, however, common

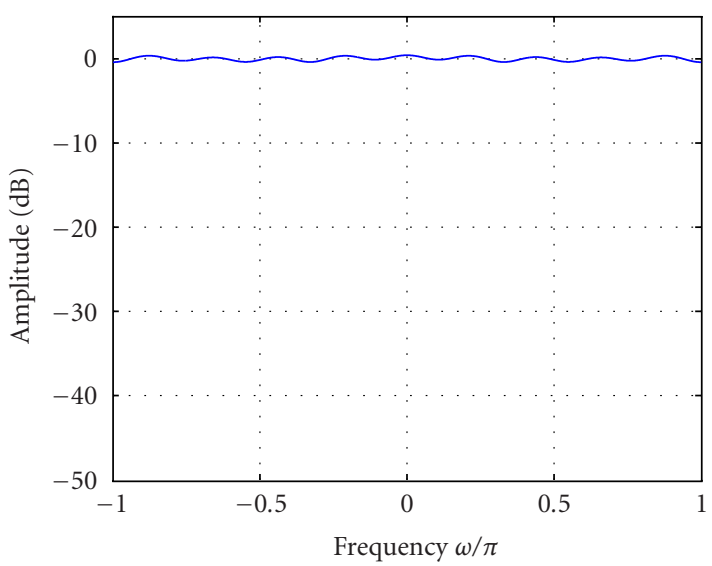

(a)

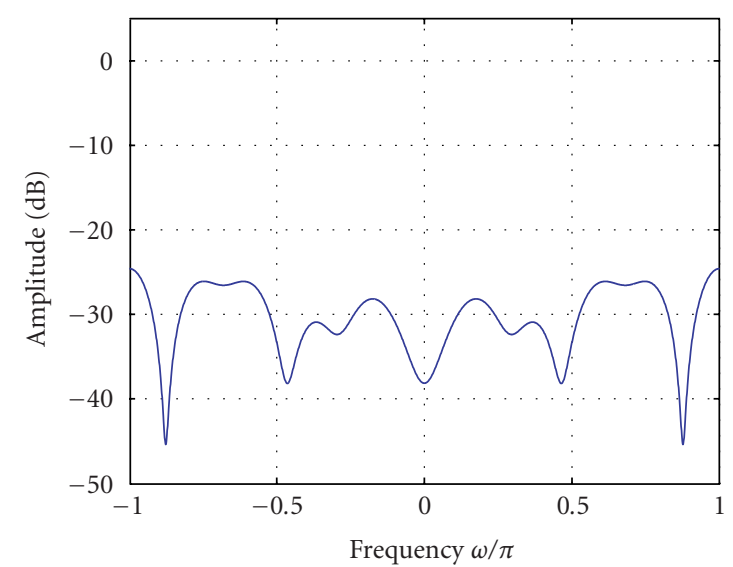

(b)

FIGURE 7: The first two components (a) $T_{1,1}(z)$ and (b) $T_{1,2}(z)$ of the total equivalent system matrix $\mathbf{T}(z)=\mathbf{W}(z) \mathbf{A}(z)$ in the steady state, the other two are not shown due to symmetry $\left(\mathbf{T}_{2,1}(z)=-\mathbf{T}_{1,2}(z)\right.$ and $\left.\mathbf{T}_{2,2}(z)=\mathbf{T}_{1,1}(z)\right)$. The separator $\mathbf{w}(z)$ is adapted using the natural gradient-based algorithm with step-size 0.001. Additive noise SNR is $20 \mathrm{~dB}$.

to all linear filtering-based techniques [6] and the signal separator-based approach is no different in this sense. Notice also that the computational complexity of any blind equalization technique is naturally higher than that of the traditional training-based solutions. However, the computational complexity of the proposed approach remains very reasonable due to the small dimension (only $2 \times 2$ ) of the used signal models, contrary to many other blind techniques reported in the literature $[14,15,16,21]$. To obtain faster convergence, separation approaches exploiting the distinct properties of individual modulation schemes, such as finite alphabet or constant modulus, can be used and constitute an interesting topic for future research.

\subsection{General discussion}

In the previous sections, the $\mathrm{I} / \mathrm{Q}$ signal models describing the effects of I/Q imbalance and carrier offsets as well as 


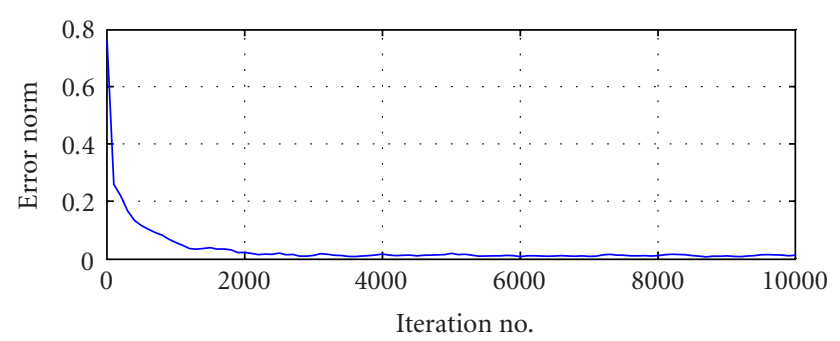

FIGURE 8: The error norm of the effective total equivalent impulse response of the equalized system with respect to a pure impulse as a function of the iteration or adaptation index. Additive noise SNR is $20 \mathrm{~dB}$.

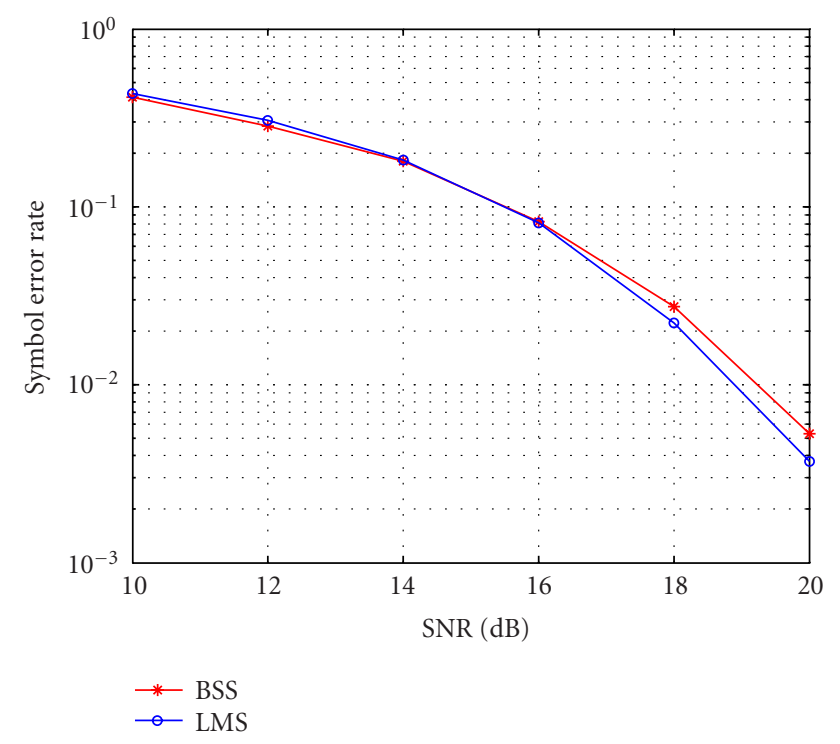

FIGURE 9: Symbol error rate of the blind I/Q signal separator-based channel equalizer (BSS) for 16-QAM data. Also shown for reference is the symbol error rate for a traditional training-based equalizer utilizing the well-known least-mean-square (LMS) algorithm.

bandpass channel dispersion were derived, resulting in instantaneous and convolutive I/Q mixtures, respectively. To simplify the notations and stay focused on the fundamentals, these two general scenarios were treated separately. Now, the generalized signal model covering all these effects simultaneously can be written as

$$
\begin{aligned}
{\left[\begin{array}{c}
x_{I}(k) \\
x_{Q}(k)
\end{array}\right]=} & {\left[\begin{array}{cc}
\cos (\Delta \omega k+\theta) & \sin (\Delta \omega k+\theta) \\
-g \sin (\Delta \omega k+\theta+\phi) & g \cos (\Delta \omega k+\theta+\phi)
\end{array}\right] } \\
& \times\left(\left[\begin{array}{cc}
h_{I}(k) & -h_{Q}(k) \\
h_{Q}(k) & h_{I}(k)
\end{array}\right] *\left[\begin{array}{c}
z_{I}(k) \\
z_{Q}(k)
\end{array}\right]\right) .
\end{aligned}
$$

The observed signals $x_{I}$ and $x_{Q}$ can thus be interpreted as instantaneous mixtures of the channel filtered I and Q data.
In other words, an adaptive instantaneous signal separator can be used to form estimates of $h_{I} * z_{I}-h_{Q} * z_{Q}$ and $h_{Q} * z_{I}+h_{I} * z_{Q}$ with the effect of (possible) instantaneous dependence due to the "zero-delay" tap $h_{I}(0)+j h_{Q}(0)$ removed. After that, a convolutive signal separator (or any other channel equalization technique) can be used to remove the dispersive mixture effects. Thus, in general, the whole structure is comprised of two parts-one accounts for instantaneous mixing and the other for convolutive effects. Given that the instantaneous "part" of the total mixing system in (23) is really time-varying (frequency offset), this cannot be avoided in general, since the order of formal multiplication $(x)$ and convolution $(*)$ in $(23)$ cannot be interchanged. However, should there be no frequency offset or it is removed by other means beforehand, (23) can indeed be written as a single convolutive type mixture model. In this case, a single convolutive I/Q separation stage can directly estimate the original I and Q data. Notice, however, that the beautiful symmetry, discussed in Section 5 (see, e.g., (20)), is in this case lost, increasing the computational complexity to some extent.

\section{CONCLUSIONS}

In this paper, blind I/Q signal separation-based approaches for receiver signal processing were proposed. More specifically, the I/Q mismatches and carrier offsets as well as the linear distortion due to general bandpass channels were shown to create crosstalk between the transmitted I and Q signals. Then compensation structures utilizing blind signal separation were used to compensate for these effects. Also some simulation results were given to illustrate the efficiency of the proposed techniques. Combining the presented I/Q mismatch and carrier offset compensation and the channel equalizer principles into a single (or a cascade of two) I/Q separator(s) results in a versatile receiver building block for future radio communication systems. Future work should be directed to further verification and prototyping of the proposed approaches using measured real-world receiver frontend signals.

Generally speaking, the idea behind this paper is to give new views for applying complex or I/Q signal processing efficiently in radio receiver design and to take full advantage of the rich signal structure inherent to complex-valued communications signals.

\section{ACKNOWLEDGMENTS}

The authors would like to thank the anonymous reviewers and the associate editor for their constructive comments and suggestions that helped significantly improve the manuscript, both in presentation and contents. This work was supported by the Academy of Finland, the Graduate School in Electronics, Telecommunications and Automation (GETA), Emil Aaltonen Foundation, Jenny and Antti Wihuri Foundation, the Finnish Cultural Foundation, and the Nokia Foundation, all of which are gratefully acknowledged. 


\section{REFERENCES}

[1] S. Mirabbasi and K. Martin, "Classical and modern receiver architectures," IEEE Commun. Mag., vol. 38, no. 11, pp. 132$139,2000$.

[2] A. A. Abidi, "Direct-conversion radio transceivers for digital communications," IEEE J. Solid-State Circuits, vol. 30, no. 12, pp. 1399-1410, 1995.

[3] J. Crols and M. S. J. Steyaert, "Low-IF topologies for highperformance analog front ends of fully integrated receivers," IEEE Trans. Circuits Syst. II, vol. 45, no. 3, pp. 269-282, 1998.

[4] J. Crols and M. S. J. Steyaert, CMOS Wireless Transceiver Design, Kluwer Academic, Dordrecht, the Netherlands, 1997.

[5] M. E. Frerking, Digital Signal Processing in Communication Systems, Chapman \& Hall, New York, NY, USA, 1994.

[6] E. A. Lee and D. G. Messerschmitt, Digital Communication, Kluwer Academic, Norwell, Mass, USA, 1988.

[7] M. Valkama, "Advanced I/Q signal processing for wideband receivers: models and algorithms," Ph.D. Dissertation, Tampere University of Technology, Tampere, Finland, 2001.

[8] G. Fettweis, M. Löhning, D. Petrovic, M. Windisch, P. Zillmann, and E. Zimmermann, "Dirty RF," in Proc. 11th Wireless World Research Forum (WWRF'04), Oslo, Norway, June 2004.

[9] M. Valkama, J. Pirskanen, and M. Renfors, "Signal processing challenges for applying software radio principles in future wireless terminals: an overview," International Journal of Communication Systems, vol. 15, pp. 741-769, October 2002.

[10] J. P. F. Glas, "Digital I/Q imbalance compensation in a low-IF receiver," in Proc. IEEE Global Telecommunications Conference (GLOBECOM '98), vol. 3, pp. 1461-1466, Sydney, NSW, Australia, November 1998.

[11] M. Valkama and M. Renfors, "Digital I/Q imbalance compensation in direct-conversion receivers," in Proc. Workshop on Software Radios (WSR '02), pp. 51-55, Karlsruhe, Germany, March 2002.

[12] I.-H. Sohn, E.-R. Jeong, and Y. H. Lee, "Data-aided approach to I/Q mismatch and DC-offset compensation in communication receivers," IEEE Commun. Lett., vol. 6, no. 12, pp. 547549, 2002.

[13] J. K. Cavers and M. W. Liao, "Adaptive compensation for imbalance and offset losses in direct conversion transceivers," IEEE Trans. Veh. Technol., vol. 42, no. 4, pp. 581-588, 1993.

[14] S. Haykin, Ed., Unsupervised Adaptive Filtering, Vol. I: Blind Source Separation, John Wiley \& Sons, New York, NY, USA, 2000.

[15] S. Haykin, Ed., Unsupervised Adaptive Filtering, Vol. II: Blind Deconvolution, John Wiley \& Sons, New York, NY, USA, 2000.

[16] A. Hyvärinen, J. Karhunen, and E. Oja, Independent Component Analysis, John Wiley \& Sons, New York, NY, USA, 2001.

[17] J. Eriksson and V. Koivunen, "Identifiability, separability, and uniqueness of linear ICA models," IEEE Signal Processing Lett., vol. 11, no. 7, pp. 601-604, 2004.

[18] J.-F. Cardoso, "Blind signal separation: statistical principles," Proc. IEEE, vol. 86, no. 10, pp. 2009-2025, 1998.

[19] J.-F. Cardoso and B. H. Laheld, "Equivariant adaptive source separation," IEEE Trans. Signal Processing, vol. 44, no. 12, pp. 3017-3030, 1996.

[20] S. Amari, S. C. Douglas, A. Cichocki, and H. H. Yang, "Multichannel blind deconvolution and equalization using the natural gradient," in Proc. 1st IEEE Workshop on Signal Processing Advances in Wireless Communications, pp. 101-104, Paris, France, April 1997.

[21] S. C. Douglas and S. Haykin, "On the relationship between blind deconvolution and blind source separation," in Proc. 31st Asilomar Conference on Signals, Systems, and Computers
(ASILOMAR '97), vol. 2, pp. 1591-1595, Pacific Grove, Calif, USA, November 1997.

[22] S. Haykin, Adaptive Filter Theory, Prentice-Hall, Upper Saddle River, NJ, USA, 3rd edition, 1996.

Mikko Valkama was born in Pirkkala, Finland, on November 27, 1975. He received the M.S. and Ph.D. degrees (both with honors) in electrical engineering (EE) from Tampere University of Technology (TUT), Finland, in 2000 and 2001, respectively. In 2002 he received the Best Ph.D. Thesis Award from the Finnish Academy of Science and Letters for his thesis entitled "Advanced I/Q Signal Processing for Wideband

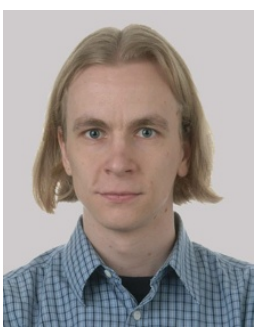
Receivers: Models and Algorithms.” In 2003, he was working as a Visiting Researcher with the Communications Systems and Signal Processing Institute at SDSU, San Diego, Calif. Currently, he is working as a Senior Researcher with the Institute of Communications Engineering at TUT, Finland. His general research interests are in communications signal processing and signal processing algorithms for flexible radios.

Markku Renfors was born in Suoniemi, Finland, on January 21, 1953. He received the Diploma Engineer, Licentiate of Technology, and Doctor of Technology Degrees from Tampere University of Technology (TUT), Finland, in 1978, 1981, and 1982, respectively. He held various research and teaching positions at TUT during the period from 1976 to 1988 . In the years 1988-1991 he was working as a Design Manager in the

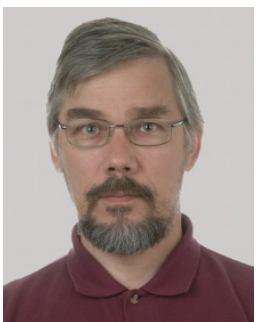
area of video signal processing, especially for HDTV, at Nokia Research Centre and Nokia Consumer Electronics. Since 1992 he has been a Professor of Telecommunications at TUT. His main research area is signal processing algorithms for flexible radio receivers and transmitters.

Visa Koivunen received his D.S. Tech. degree with honors from the Department of Electrical Engineering, University of Oulu, and was selected the Primus Doctor (Best Graduate) in years 1990-1994. From 1992 to 1995 he was a Visiting Researcher at the University of Pennsylvania, Philadelphia, USA. In 1996 he held a faculty position at the Department of Electrical Engineering, University of Oulu. From August 1997 to August 1999 he was an Associate Professor at the Signal Processing Laboratory, Tampere University of Technology. Since 1999 he has been a Professor of signal processing at the Department of Electrical and Communications Engineering, Helsinki University of Technology (HUT), Finland. He is one of the Principal Investigators in SMARAD Center of Excellence in Radio and Communications Engineering nominated by the Academy of Finland. In 2003 he was appointed to Adjunct Professor at the University of Pennsylvania, Philadelphia, USA. Dr. Koivunen's research interests include statistical and sensor array signal processing as well as wireless communications. He has published more than 160 papers in international scientific conferences and journals. He is a Member of the IEEE Signal Processing for Communication Technical Committee (SPCOM-TC). 